\title{
Neuronal avalanches recorded in the awake and sleeping monkey do not show a power law but can be reproduced by a self-organized critical model

\author{
Viola Priesemann*1, Michael Wibral ${ }^{2}$ and Matthias HJ Munk ${ }^{3}$
}

\author{
Address: ${ }^{1}$ Max Planck Institute for Brain Research, 60528 Frankfurt, Germany, ${ }^{2}$ MEG Unit, Brain Imaging Center, Goethe University, 60528 \\ Frankfurt, Germany and ${ }^{3}$ Max Planck Institute for Biological Cybernetics, 72076 Tuebingen, Germany \\ Email: Viola Priesemann* - v.priesemann@gmx.de \\ * Corresponding author
}

from Eighteenth Annual Computational Neuroscience Meeting: CNS*2009

Berlin, Germany. 18-23 July 2009

Published: I 3 July 2009

BMC Neuroscience 2009, I0(Suppl I):PI6I doi:I0.II86/|47|-2202-|0-SI-PI6I

This abstract is available from: http://www.biomedcentral.com/|47|-2202/I0/SI/PI6I

(C) 2009 Priesemann et al; licensee BioMed Central Ltd.

Self-organized critical (SOC) systems are complex dynamical systems that may express cascades of events, called avalanches [1]. The SOC state was proposed to govern brain function, because of its activity fluctuations over many orders of magnitude, its sensitivity to small input and its long term stability $[2,3]$. In addition, the critical state is optimal for information storage and processing [4]. Both hallmark features of SOC systems, a power law distribution $f(s)$ for the avalanche size $s$ and a branching parameter (bp) of unity, were found for neuronal avalanches recorded in vitro [5]. However, recordings in vivo yielded contradictory results [6]. Electrophysiological recordings in vivo only cover a small fraction of the brain, while criticality analysis assumes that the complete system is sampled. We hypothesized that spatial subsampling might influence the observed avalanche statistics. In addition, SOC models can have different connectivity, but always show a power law for $f(s)$ and $b p=1$ when fully sampled. This may not be the case under subsampling, however. Here, we wanted to know whether a state change from awake to asleep could be modeled by changing the connectivity of a SOC model without leaving the critical state.

We simulated a SOC model [1] and calculated $f(s)$ and bp obtained from sampling only the activity of a set of $4 \times 4$ sites, representing the electrode positions in the cortex. We compared these results with results obtained from multielectrode recordings of local field potentials (LFP) in the cortex of behaving monkeys. We calculated $f(s)$ and bp for the LFP activity recorded while the monkey was either awake or asleep and compared these results to results obtained from two subsampled SOC model with different connectivity.

$f(s)$ and bp were very similar for both the experiments and the subsampled SOC model, but in contrast to the fully sampled model, $f(s)$ did not show a power law and bp was smaller than unity. With increasing the distance between the sampling sites, $f(s)$ changed from "apparently supercritical" to "apparently subcritical" distributions in both the model and the LFP data. $f(s)$ and bp calculated from LFP recorded during awake and asleep differed. These changes could be explained by altering the connectivity in the SOC model.

Our results show that subsampling can prevent the observation of the characteristic power law and bp in SOC systems, and misclassifications of critical systems as sub- or supercritical are possible. In addition, a change in $f(s)$ and bp for different states (awake/asleep) does not necessarily imply a change from criticality to sub- or supercriticality, but can also be explained by a change in the effective connectivity of the network without leaving the critical state. 


\section{Acknowledgements}

We want to thank J. Klon-Lipok for help with data acquisition and M. Beian for help with data preproceessing.

\section{References}

I. Bak P, Tang C, Wiesenfeld K: Self-organized criticality: An explanation of the I/f noise. Phys Rev Lett 1987, 59:38I-384.

2. Bak P: How nature works: the science of self-organized criticality New York: Copernicus Press; 1996.

3. Jensen HJ: Self-Organized Criticality Cambridge University Press, Cambridge, UK; 1998.

4. Bertschinger $\mathrm{N}$, Natschläger $\mathrm{T}$ : Real-time computation at the edge of chaos in recurrent neural networks. Neural Comput 2004, 16: | 413-1436.

5. Beggs JM, Plenz D: Neuronal avalanches in neocortical circuits. J Neurosci 2003, 23: I I 167-III I77.

6. Bedard C, Kroeger H, Destexhe A: Does the I/f frequency scaling of brain signals reflect self-organized critical states? Phys Rev Lett 2006, 97:118102.

Publish with Bio Med Central and every scientist can read your work free of charge

"BioMed Central will be the most significant development for disseminating the results of biomedical research in our lifetime. "

Sir Paul Nurse, Cancer Research UK

Your research papers will be:

- available free of charge to the entire biomedical community

- peer reviewed and published immediately upon acceptance

- cited in PubMed and archived on PubMed Central

- yours - you keep the copyright

Submit your manuscript here:

http://www.biomedcentral.com/info/publishing_adv.asp 